\title{
Electronic and computer monitoring in shock
}

\author{
JOHN C. A. RAISON \\ From the Department of Health and Social Security, London
}

Instrumental means of measurement and monitoring extend human observations in the care of shocked patients. Computers stretch still further the capacity to measure and to process information. Apart from the more technical applications to be discussed in this paper, it should be mentioned that modern computer installations have the proven ability to collect, collate, and present effectively data from multiple sources, a task which becomes progressively more difficult and unduly demanding as a result of the therapeutic requirements for more measurements and the use of many separate pieces of equipment to treat a single patient. One computer system is devoted exclusively to this (Norlander, Mellberg, Pettersson, and William-Olsson, 1968; Mellberg, 1968) and such handling of information is a significant part of others (Osborn, Beaumont, Raison, Russell, and Gerbode, 1968). Circumstances of increasingly intense and scientific care in the face of diminishing personnel resources will promote this development. A number of the free-standing monitoring devices which are already available incorporate purpose-built analogue or digital processing logic. Several research and development projects for intensive therapy now make use of computer facilities to obtain measurements not otherwise obtainable in the special clinical situation, to provide new measuring devices, to aid in calibration and maintenance of equipment, or to participate in the control of treatment. Such systems raise the important consideration of cost-benefit analysis and concentration of patients receiving such care to justify such facilities, but these are ignored here in the interest of searching for the highest achievable level of clinical attention. It is intended to consider as one the uses of electronic and computer techniques, ranging over a number of physiological functions or required measurements. Because progress is fast in this leading edge of medical care, it is necessary to mention research developments which will or may shortly be practicable in treatment, whilst discussing present resources, but a distinction will be drawn between the two.

\section{Cardiovascular Measurements}

Unless there is any preexisting associated pathology, cardiac abnormalities in traumatic shock will usually only arise as relatively late manifestations of anoxia or hypovolaemia. The critical exception is the occurrence of cardiac tamponade. The correlation of trends in the measurements of arterial and venous pressures, cardiac output, electrocardiogram results, and some other indices of atrial or ventricular function form the basis of diagnosis. Perhaps the most contributive, and least developed, monitoring function of digital computers is the application of the logical examination of multivariate trends and the presentation of data in fairly precise diagnostic words, rather than as numbers for analysis and interpretation. At least one such experimental program has been outlined for tamponade (Osborn, Beaumont, Raison, and Abbott, 1969a) and we should hope for clinically applicable derivatives. It is a familiar problem of ECGmonitoring apparatus that false alarms are so frequent as to cause most rate-limit indicators to be rendered inoperable: this is overcome in more recent apparatus using local logic systems or by correlating ECG and vascular waveform signals (Raison, Beaumont, Russell, and Osborn, 1968b).

There is now widespread experience that the measurement of intraarterial pressure by percu- 
taneous puncture is a safe, desirable, and relatively simple means of securing a reliable blood pressure reading in shocked patients. Some commercial monitoring modules already provide digital displays of values, including pulse pressure which is as important a sign in shock as the more commonly accepted systolic and diastolic readings. One such monitoring system provides a data-recording system. A major advantage of such apparatus, or computer installations, is this provision which ensures the continuation of data logging in periods of crisis when it can be most significant but most likely to be abandoned. It has also been observed that traditional annotation may produce records that are inaccurately related to time and may be misleading (Osborn et al, 1969a).

The first derivative of arterial pressure, obtained by differentiation of the waveform upstroke, reflects functions of left ventricular performance and peripheral resistance: the latter may be of particular importance in cases of trauma where septic shock has supervened. At present there is insufficient knowledge of the characteristics of change in these signs to be of practical use in therapy, but the vastly improved facilities for detailed clinical research on shocked patients, without detriment to them, provided by the recording and analytical powers of computerbased systems should lead to rapid developments of direct value in caring for patients. Although there are still shortcomings in pressure transducers, considerable progress has been made which permits their routine use. Already there is a 'small' transducer which can be strapped close to the site of skin puncture, thereby minimizing nursing problems associated with movement and risks of accidental blood loss from cannula disconnexion. Autoclavable transducers will shortly be available. Transducers with drift characteristics of less than $1 \mathrm{~cm}$ water over 24 hours in a range of normal ward temperatures and stable sensitivity, which permit long-term measurement without heavy demands for attention, are now available. On-line computer systems already apply simple logic checks, such as identification of arterial catheter damping. Correlation with ECG readings to eliminate false alarms has previously been mentioned.

The measurement of central venous pressure, which provides information about effective circulating blood volume and heart failure, usually involves repeated manual steps and observation. Straightforward application of a pressure transducer for continuous automated measurement brings its own problems. The most commonly accepted reference point for such readings, approximating to the right atrial position, makes the application of the transducer to the skin difficult or impossible; postural changes therefore require realignment of the transducer. By means of a second transducer connected to a postural position sensor which may be fixed close to the axilla, automatic reading of centre? venous pressure (the subtraction of position a pressure from observed reading of venous tran ducer) is possible. A computer-based system has provided for checking, measurement, and pos tional calculation, as well as permitting intras venous infusion between readings (Raisorg Beaumont, Shore, and Osborn, 1968c).

As methods become more easily applied the estimation of cardiac output will contribute more frequently to care. Instrumentation for dye dilution studies now embodies logic and calculas tion to produce digital readout, eliminating the tedium of extrapolation of the downslope and integration of the primary curve. There remaing some doubt whether the original Hamiltog method of estimation (Hamilton, Moore, Kins; man, and Spurling, 1932) is reliable in low output states. Methods based on the forward edge of the dye curve (Dow, 1955) may be preferable. Agaifin we are in the period when computer monitoring systems capable of multiple correlated measurements by various methods and using various formulae should rapidly accumulate data and produce evidence from which the most effective method for any particular clinical situation may be deduced. It may be that estimation using different formulae for different clinical states or comparison of two or more methods of estimation, is desirable for optimum care. Ore computer system provides two calculations, and has fully automated the test.

Although not yet wholly acceptable, the estimation of change in cardiac output based upon arterial wave upstroke (ejection charae teristics) offset by calculations for run-off through the arterial bed (peripheral resistance), as devised by Warner, Gardner, and Toronto (1968), may be of use. However, there remains the possibilify that changes in peripheral resistance during the course of shock, especially shock due to sepsis; may limit the reliability of this method. Intraluminal flow measurement may eventually be the method. Estimations based on the Fick calcul tion, from arteriovenous oxygen difference and oxygen uptake, can be criticized as this method relies too heavily on a steady state. In practige such estimations have correlated well with other estimations in at least one low output situation after open-heart surgery.

Blood volume measurements are not widery judged to be valuable in shock treatment, although instruments for radioactive scintillatiơn counting are now extremely simple to use. This reservation is due less to doubts about the clinical effectiveness of relying on central venooss pressure measurement for replacement therapy than to doubts about the appropriate equilibestion intervals after injection of the tracer dose gr the rate of exchange between body compartments.

In situations of trauma where volume measufment might most be wanted, as for instance in cases of burns, the measurement is even moje 
likely to be unreliable. With the move towards treatment based on precise measurements, it is to be expected that the potential of on-line computers to permit the use of multiple tracer techniques to measure the exchange between intra- and extra-vascular spaces in patients in shock, without immense and time-consuming quantities of data to calculate 'manually', will be exploited to provide useful direct methods of volume determination. It is another advantage of a computer-based system that it allows calculation of the radioactive background and of the next minimal dose required for significant measurement in any series of such investigations. Output and volume estimations may be made from a single procedure.

\section{Temperature}

The existence of shock justifies the disadvantages of a rectal temperature probe for continuous measurement. In time, multiple function intravascular catheters are to be expected (pressure, flow, temperature, as well as sampling lumen). It may be found that a central venous thermistor probe is a more useful device. Core temperature observation is important to assess metabolic rate and oxygen demand, but it has greater significance when combined with several skin temperature probes which are usually taken on the lower limbs. In the present crude state of knowledge, changes between skin temperature and core temperature readings are meaningful as a reflexion of cardiac output and peripheral circulation in shock. Another probe should measure environmental temperature, so that skin temperature may be related to heat loss by radiation. Whilst useful information about the improvement or deterioration of the circulation (and by inference about cardiac output) is obtainable from a simple display of increasing warmth or cooling of the limbs, it should be possible from a computer-based system to establish far more precise relationships, probably related to several different points along a limb. In a fully integrated monitoring system which uses the minimum of penetrative techniques possible, temperature characteristics may be one of the most valuable clinical signs.

\section{Blood Gas Measurements}

Standard electrode instrumentation for the measurement of $\mathrm{PO}_{2}, p \mathrm{H}$, and $\mathrm{PCO}_{2}$ (either directly or by the interpolation method of Astrup) are now very reliable and should form part of the stock in trade of any unit treating shock. It is preferable that they should be performed at $37-38^{\circ} \mathrm{C}$ and questionable whether in view of the wide range of temperature in the whole body, 'corrections' to observed body tem- perature are appropriate, unless clinical hypothermia exists. Metabolic acidosis in shock is pathognomonic of low cardiac output, and $\mathrm{PCO}_{2}$ measurements, if done sufficiently often, can effectively monitor hyper- or hypoventilation. $\mathrm{PO}_{2}$ measurements are also important in monitoring ventilation and respiration. For most reliable arteriovenous difference estimations it is better to combine the use of oxygen tonometry and $p \mathrm{H}$ measurements to determine arterial oxygen content, with oximetry to determine venous content. A commercially available oximeter will probably be the normal equipment. Sometimes two wavelength oximeters are used (Eberhart, 1968) and their development would be helpful. With these the measurement at the isobestic frequency gives a reading proportional to haemoglobin content, which otherwise requires separate determination. A number of systems have been provided which render blood gas measurements on-line, in the sense of a direct connexion from patient cannula to machine. Sample collection, calibration, measurement, calculation, and output are all automated. The economics of necessarily dedicating such apparatus to a single patient suggest the limitations of such a concept. Electrodes for use in vivo by intravascular insertion are now appearing, and, since it is possible to connect a number coming from different patients to a single electronic measuring unit, costs may not be too great. There is not yet sufficient clinical experience to be sure that protein deposition on electrodes will not alter their responses significantly over prolonged periods in situ. One of the advantages of such a system is the notable saving in the volume of the sample withdrawn from the patient. It remains to be established that continuous reading of blood gases and $p \mathrm{H}$ has any clinical advantage over frequent intermittent measurements. Another expensive tool which is being developed and estimates $\mathrm{PO}_{2}$ and $\mathrm{PCO}_{2}$ in the blood is the use of a closed plastic-tipped (gas-permeable), highvacuum cannula attached to a mass spectrograph. With this continuous measurement is again possible. It is unlikely that any of these more recent developments will come into common clinical use, even in shock units, for several years yet.

In units where a computer is available, on-line connexion to it brings advantages which will be more fully discussed later. At the technical level, frequent analyses can be called for by nurses or house staff who have only to inject samples into a bed- or ward-side machine. Such machines require about 30 minutes a day for a trained technician to maintain.

\section{Blood Electrolytes}

The measurement of blood electrolytes is less advanced than blood gas measurement; specific- 
ally permeable electrodes for measuring sodium and potassium are commercially available. These models require considerably more skill in use and maintenance. The calculation of potassium is dependent on the sodium value found. As well as these, a semi-automatic blood analyzer has been produced, in which a single blood sample $\left(\mathrm{PO}_{2}, \mathrm{PCO}_{2}, p \mathrm{H}\right.$, sodium, and potassium) is examined by a series of electrodes (Osborn et al, 1968; Widdowson, G., Pacific Med. Ctr., personal communication). Again, the whole process, including calibration with two reference gases and solutions, is automated after introducing the sample. Most of the logic for the system, including multiplexing of electrode readings to a meter, is internal to the machine so that it has a stand-alone capability. However, on-line connexions to a computer are used to process analogue signals, and to make necessary corrections for interrelated variables and temperature before providing printed output or values for further correlation with other physiological signals processed by the system. Technical attention is again limited to about 30 minutes daily. It is possible that a chloride $\left(\mathrm{Ag} / \mathrm{AgCl}_{2}\right)$ electrode may also become of practical use. All these methods of electrolyte determination have been largely confined to research-oriented units. It is unlikely that their use as monitoring apparatus, close to the patient, is frequently called for in conditions of traumatic shock in a previously healthy population. The measurements required, at longer intervals of time, can for the moment be more easily made in the standard clinical chemistry laboratory. At present their relevance in non-cardiogenic shock is largely to act as indicators of a return towards normal homeostasis following the more immediate corrections of low cardiac output or renal failure. Again, however, an increase in accumulation of clinical data and analysis of short term electrolyte shifts, probably using labelled electrolytes, associated with the availability of computers, may lead to therapeutic plans which require much more frequent, and hence bedside, measurement.

\section{Monitoring of Ventilation and Respiration}

A number of simpler instruments measure respiratory rate using the change of airway temperature (from a nasal thermistor probe) or recorded change in strain gauges attached to the chest. Their usefulness in monitoring shock is limited and they have not been widely adopted. Impedance plethysmography can supply rate values, and it is possible to determine tidal volume if signals can be calibrated against a known volume change in the individual patient. However, especially in shock, changes in the flow of pulmonary blood, and variations in zonal ventilation have not yet been evaluated and the method is not yet clinically applicable.
A very considerable proportion of shocke patients require mechanical ventilation, and there is reason to believe that better monitoring of ventilation and respiration will contribute muन्तु to improving their care. Recently it was found that in eight patients developing 'unexplain $\overline{\text { d }}$ cardiac crisis', five of the crises were directby attributable to ventilatory inadequacies begiōning up to several hours previously, all detectab by a computer-based monitoring system but nबi detected by other means in a well staffed unit of highly trained nurses (Osborn, Raison, Beaumonूt Hill, Kerth, Popper, and Gerbode, 1969b. The fewest measurements required are: (ㄱ) ventilatory rate, tidal volume, and minu依 volume; (2) airway pressure; (3) concentration $\frac{\mathrm{g} f}{\mathrm{f}}$ inspired oxygen; and (4) end-tidal carbon dioxide concentration.

Volume changes obtained from spirometers in ventilators, or inserted in the airway, are subjecy to many errors associated with variable gas flosv rates, compression and leakage of gases. Two of the ventilatory inadequacies mentioned above were related to an unexplained sudden change in the tidal volume delivered, or undetected leaks in corrugated tubing. Airway pressure is an important indication of progressive obstruction or change in pulmonary compliance: gauges attached to ventilators provide very coarse and unreliable measurements.

Oxygen concentration may be measured by airway polarographic electrode, or by a paro: magnetic analyzer in a small parallel airway loog. Prolonged ventilation with $100 \% \mathrm{O}_{2}$ is associate with progressively larger alveolar arterialoxyge gradients and is to be avoided when possible. Flow valve mixing of supply gas to ventilators can be unreliable as a means of determining con: centration, and under certain conditions (Pontoppidan and Berry, 1967) pressure-triggered ven $\bar{c}$ lators are now well known to supply very mue higher $\mathrm{O}_{2}$ concentrations than intended. End tidal carbon dioxide concentration reflects $\mathrm{PCG}_{2}$ and the adequacy of ventilation. The making and recording of all these measurements is extremety demanding on the nursing staff.

Methods have now been developed to provide all these data, and a number of other measurements, that may be desirable or potentially usefun automatically on a computer-based monitoring system. As well as making the necessary calcula tions, displaying and recording results, importamt correlations with other measurements are made and some diagnostic logic is applied. Details have been published (Beaumont, Osborn, Raison, and Russell, 1968; Raison, Beaumont, Elliott, arod Osborn, 1968a; Osborn et al, 1968 and 1969b; Osborn, Elliott, Segger, and Gerbode,1969c) ang only a brief description is possible here.

A pneumotachograph placed in line as close to the patient's trachea as possible provides differential signal: after digital conversion the computer integrates instantaneous flow rates 
provide volumetric data. Because of alarm requirements the sampling for analysis of flow and airway pressure is continuous. The pneumotachograph is further modified by welding in a fine-bore sampling line. During intervals of analysis, 30 seconds in every 10 minutes, or when demanded by ward staff, airway gas is aspirated at the slow rate of $1.5 \mathrm{l} / \mathrm{min}$, carried to the bedside monitoring console and there divided between a rapid infra-red $\mathrm{CO}_{2}$ analyzer and an even faster response hot platinized zirconium oxide oxygen electrode. Both these are fast enough to follow gas concentrations throughout the inspiratory-expiratory cycle. Between analyses warm dry air is blown in the reverse direction down sampling and pressure lines to the pneumotachograph to eliminate condensation. By this means the system remains in situ for well over 24 hours at a time without attention. The computer also provides compensatory gas withdrawal during these blow-back periods so that the characteristics of ventilation are not different during or between analysis periods. In the present arrangement, each pair of gas analyzers serves two patients alternatively, and seven patients can be monitored simultaneously.

The system already provides semi-automated calibration of all the measuring instruments used. It merely requires a technician, checking once daily, to apply the pneumotachograph to an apparatus simulating inspiration and expiration in the airway, and to push a piston to and fro for several rapidly sequenced periods of analysis whilst checking the displayed results with those required from this dummy system. Fully automated calibration of all measurements should be possible in future systems.

A wide range of calculations, correlations, and diagnostic analyses is then performed. Output is directed to visual data terminals at the bedside, with verbal interpretation of the values shown whenever a diagnostic identification is made. Output is also directed to a teletypewriter in the ward for permanent revised storage and to an incremental X-Y plotter to provide charts for daily clinical ward rounds. Disc storage permits retrieval of old data and comparisons on request at the terminal for up to four or five days. The system similarly processes and records all other data that have been acquired, some of which are used in correlations.

The first clinical application occurs when connecting patients to ventilators. On push button command, a continuous analysis is started, which provides a breath-by-breath indication of rate and tidal volume; ventilator adjustments are much simplified and more accurate. A graphic display of the inspiratory portion of each breath cycle is produced which is of great value in most satisfactorily manipulating ventilatory characteristics. This facility is also used for regular monitoring of chest compliance and the need for airway toilet, and has highlighted advantages over the previous method, largely confined to the sort of nursing instruction as 'suck out airway every $x$ minutes'.

Alarm limits are set on pneumotachograph parameters to identify apnoea, sudden changes in volume or rate, leakage, and changes in chest compliance or non-elastic resistance. Ordinary results are displayed. Mean inspired oxygen concentration is shown, and end-tidal $\mathrm{CO}_{2}$ to indicate hyper- or hypoventilatory changes. Alveolar ventilation is calculated: it was a not uncommon experience that the spirometer reading on a volume-cycled ventilator was very grossly in excess of the measured tidal and alveolar volumes. Alveolar oxygen concentration is computed at each analysis. Whenever blood gas data become available from the on-line analyzer, or from keyboard entries, the data log is searched and the two ventilatory analyses most adjacent in time are used to produce a number of interrelated factors, including alveolar-arterial $\mathrm{O}_{2}$ 윽 gradient and the physiological dead space to tidal volume ratio. The availability of these estimations without elaborate measurement and calculation has led to a much more precise supervision of ventilation.

Oxygen consumption is also calculated. The computer integrates the product of instantaneous oxygen concentration and tidal volume observations: inspiratory and expiratory values for the period are averaged and the latter is subtracted from the former. Five additional steps then take place which diminish the errors due to changes between observed inspired and expired volumes etc (Raison et al, 1968a; Osborn et al, 1969a). Expiration of $\mathrm{CO}_{2}$ is similarly estimated. If a Fick estimation of cardiac output is requested by simple command, the system links arterial and venous blood analyses with an automatically instituted three-minute estimation of oxygen uptake. If a dye-dilution estimation of cardiac output is started, together with arterial and venous samples, a Fick calculation will also be carried out and the results displayed beside the other estimation.

It was hoped that monitoring of oxygen uptake in shock might provide a sensitive sign of deterioration, possibly even having a quantitative predictive value, if human pathophysiology followed the oxygen deficit pattern decribed by Crowell and Smith (1964) in animals. This does not appear to be so (Raison, Osborn, Beaumont, $\varphi$ and Gerbode, 1968d). The clinical usefulness of $\Phi$ this method has been to illustrate the disturbing $?$ and very large increased oxygen demand caused by restlessness and shivering. Sometimes this increased uptake has been the first pointer to an almost unnoticeable fine fibrillary muscular twitching, which, when controlled by relaxants, produced a satisfactory return to more normal uptake.

There are also interesting possibilities of therapeutic importance in the observation of 
'abnormal' oxygen uptake in response to the use of isoprenaline (Raison et al, 1968d) which may reflect important changes in the peripheral circulation, and in changes of respiratory quotient which may render this form of monitoring of greater importance in the future than it is today.

An alternative method of estimating the composition of ventilatory gas is by the use of small-mass spectrometers now being produced. This is not yet in practice anywhere, but preliminary tests show that a breath-by-breath response can provide the computer with adequate signals. It is not clear whether there are advantages in such a method, except when explosive gases or halothane are used (the hot ceramic oxygen electrode is heated to $800^{\circ} \mathrm{C}$, and halothane causes a strange biphasic response-100\% oxygen does not present a hazard). Spectrometry may be a more expensive method.

Three other pieces of information which are very necessary may soon be available from such systems. The inspiratory phase of the pressurevolume ventilation loop, which is at present reviewed by staff, is clearly composed of three elements. First, inspiration commences. When a pressure-cycled ventilator is used a deflexion of pressure indicating undue suction may be detected. The second, and succeeding element, is the main period of gas inflow: the pressurevolume change here most directly reflects pulmonary compliance. The final phase, which appears as a small hook on the graph, indicates the cut-off point for inspiration and the work which may have to be done to cause gas flow in reverse direction. All these have different characteristics in various undesirable states. Curvefitting procedures are being developed for each phase which should provide far more precise description of the 'diagnosis' in verbal terms rather than the presentation of raw data made by the computer (Miller and Osborn, quoted in Osborn et al, 1969b). The second potential is that the true arterial $\mathrm{PCO}_{2}$ may be calculable from the expiratory curve for $\mathrm{CO}_{2}$ combined with the total $\mathrm{CO}_{2}$ production. And the third, that pulmonary blood flow may also be calculable. These achievements might greatly diminish the number of patients in whom arterial penetration is needed. It is worth noting, however, that all the data discussed in this paper can be obtained from only two, relatively common, penetrations for arterial and venous cannulae.

\section{Computers in Monitoring}

It may be useful here to review separately the part computers play in this situation. Apparatus designed for one or two measurements only may include analogue or digital logic to produce a set of information (eg, cardiac output, or the more expensive ECG monitors). When a well established array of required measurements and displays has been defined, if such agreement can ever be obtained, there may be a logical develop ment of this principle in the provision of purpose built computers for such requirements, using cheaper fixed wire logic. This may be an economic solution for the future.

A fairly small scientific processor (perhate only $4 \mathrm{~K}$ store and without fast remote access such as disc or drum) might be used as an add tional instrument for specific tests (ventilator. cardiac output, ECG analysis) on the basis of reprogramming it (probably by paper tape insere) for each required activity in turn. The cost mig be $£ 10,000$ to $£ 20,000$. The limiting factor is the feasibility of repeatedly changing programs. Sus a system probably intensifies the problems off multiple discrete pieces of data record and the correlation.

Finally, a comprehensive system can acquire analog and digital data, carry out on-line estimas tions in real time, calibrate and monitor the tests themselves and pre-set alarm limits, perform required correlations, and make logicaldiagnostic interpretations. Trend analysis of multivariase data is already being developed by researef teams (another step nearly impossible in cases of shock without comprehensive processing facilit ties) which should greatly enhance the use of data. True process control (sometimes called 'closing the loop') in intensive care is possible. Although in spite of the reassurance of 'manu氛 override' some are chary of a machine direct instituting treatment two monitoring systen (Birmingham, Ala. and Rochester, Minn? already incorporate intravenous infusion contro and appear to be more accurate than huma control for both blood and the use of vasoacti drugs.

A computer-based system can provide displar and data records as required. An important feature is the retention of flexibility which permits. rapid and simple steps to change the correlations of data or displays which the clinician inevitabl requires as progress in care develops fast. The advantages such a system has in logging data cam produce greater accuracy and vastly increase time the nursing staff can devote directly to their: patients. The cost may be from $£ 50,000$ to we over $£ 100,000$. Multiplexing of considerably more than six beds is possible. Economics seen to dictate that this sort of system, probably the best, requires the bringing together of patients needing such care, who have sometimes becone scattered in various units throughout a hospita including the operating suite; such collection may, of course, be by wired data links, althoug costs rise again. Perhaps several hospitals shoug be served by one system.

\section{Conclusion}

Instrumentation now plays an important role 
the care of all patients in shock. As it increases in availability, the initial advantages are progressively offset by the greater demands made on staff in terms of time spent in recording and reviewing multiple discrete signals, or in calibration and maintenance by special skilled staff. A problem of how to place large quantities of apparatus around the patient and still provide access for the nurse also arises. Computer systems have capabilities of increasing the measurements which may be made safely and conveniently in shock; of automatic calibration; increasing accuracy of some measurements; sharing of instrumentation; making calculations of value which would otherwise not be undertaken in practical therapy; of correlations, logical checking, diagnosis, and advanced method of data display and recording in terms most useful to the ward team. There are complex economic consequences which must affect the speed of development. Measurements required and functional systems which need monitoring have been reviewed in the light of instrumental and computer facilities available today or anticipated.

Most of the clinical experience discussed here was developed with the collaboration of colleagues, Drs J. J. Osborn, J. O. Beaumont, and F. Gerbode, and others, of the Pacific Medical Center, San Francisco, aided by USPHS grant HE:06311: it is very warmly recognized.

\section{References}

Beaumont, J. O., Osborn, J. J., Raison, J. C. A., and Russell, J. A. G. (1968). Respiratory measurement and monitoring based on an on-line computer. Proceedings of the Conference of the Association for the Advancement of Medical Instrumentation, Houston, Texas.
Crowell, J. W., and Smith, E. E. (1964). Oxygen deficit and irreversible hemorrhagic shock. Amer. J. Physiol., 206, 313-316.

Dow, P. (1955). Dimensional relationships in dye dilution curves from humans and dogs with an empirical formula for certain troublesome curves. J. appl. Physiol., 7, 399-408.

Eberhart, R. C. (1968). An automated sampling whole blood photometer. Biomed. Sci. Instrum., 4, 197-204.

Hamilton, W. F., Moore, J. M., Kinsman, J. N., and Spurling, R. G. (1932). Studies on circulation. Amer. J. Physiol., 99, 534-551.

Mellberg, K. (1968). Integrated data display system for patient monitoring at the Caroline Hospital. Proc. 21st ACEMB, 17,8 .

Miller, C. W., and Osborn, J. J., quoted in Osborn et al, 1969b.

Norlander, O., Mellberg, K., Pettersson, S. O., and WilliamOlsson, G. (1968). An integrated display system for patient-data monitoring. (Excerpta med. int. Congr. Ser., 168), p. 18. Excerpta Medica Foundation, Amsterdam.

Osborn, J. J., Beaumont, J. O., Raison, J. C. A., Russell, J. A. G., and Gerbode, F. (1968). Measurement and monitoring of acutely ill patients by digital computer. Surgery, 64, 1057 . 1070.

Osborn, J. J., Beaumont, J. O., Raison, J. C. A., and Abbott, R. P. (1969a). Computation for quantitative on-line measurements in an intensive care ward. In Computers in Biomedical Research, edited by R. W. Stacy and B. Waxman, vol. 3, pp. 207-237. Academic Press, New York.

Osborn, J. J., Raison, J. C. A., Beaumont, J. O., Hill, J. O., Kerth, W. J., Popper, R. W., and Gerbode, F. (1969b). Respiratory causes of 'sudden unexplained arrythmia', in post thoracotomy patients. 22nd Conf. Amer. Heart Ass., Scientific Sessions.

Osborn, J. J., Elliott, S. E., Segger, F. J., and Gerbode, F. (1969c). Continuous measurement of lung mechanics and gas exchange in the critically ill. Med. Res. Eng., 8, 19-23 and 32.

Pontoppidan, H., and Berry, P. R. (1967). Regulation of the inspired oxygen concentration during artificial ventilation. J. Amer. med. Ass., 201, 11-11.

Raison, J. C. A., Beaumont, J. O., Elliott, S. E., and Osborn, J. J. (1968a). Breath-by-breath analysis of respiratory gases in acutely ill patients. Proc. 21st ACEMB, 2.

Raison, J. C. A., Beaumont, J. O., Russell, J. A. G., and Osborn, J. J. (1968b). Alarms in an intensive care unit: an interim compromise. Computers biomed. Res., 1, 556-564.

Raison, J. C. A., Beaumont, J. O., Shore, J., and Osborn, J. J. 1968c). Automated measurement of central venous pressure by on-line real-time computer method. Proc. 21st $A C E M B, 4,9$.

Raison, J. C. A., Osborn, J. J., Beaumont, J. O., and Gerbode, F. (1968d). Oxygen consumption after open heart surgery measured by a digital computer system. Ann. Surg., 171, 471-484.

Warner, H. R., Gardner, R. M., and Toronto, A. F. (1968), Computer-based monitoring of cardiovascular functions in postoperative patients. Circulation, 37, Suppl. 2, 68-74. 